\title{
Electronic health record interventions at the point of care improve documentation of care processes and decrease orders for genetic tests commonly ordered by nongeneticists
}

\author{
Maren T. Scheuner, MD, MPH'1,2, Jane Peredo, ScM¹, Kelly Tangney, MS1', Diane Schoeff'1, \\ Taylor Sale, MS1 ${ }^{1}$, Caroline Lubick-Goldzweig, MD, MSPH ${ }^{1,2}$, Alison Hamilton, PhD, MPH ${ }^{1,3}$, \\ Lee Hilborne, MD, MPH${ }^{4,5}$, Martin Lee, PhD', Brian Mittman, PhD', Elizabeth M. Yano, PhD, MSPH ${ }^{1,6}$ \\ and Ira M. Lubin, $\mathrm{PhD}^{7}$
}

Objective: To determine whether electronic health record (EHR) tools improve documentation of pre- and postanalytic care processes for genetic tests ordered by nongeneticists.

Methods: We conducted a nonrandomized, controlled, pre-/postintervention study of EHR point-of-care tools (informational messages and template report) for three genetic tests. Chart review assessed documentation of genetic testing processes of care, with points assigned for each documented item. Multiple linear and logistic regressions assessed factors associated with documentation.

Results: Preimplementation, there were no significant site differences $(P>0.05)$. Postimplementation, mean documentation scores increased $(5.9(2.1)$ vs. $5.0(2.2) ; P=0.0001)$ and records with clinically meaningful documentation increased (score $>5$ : 59 vs. $47 \% ; P=0.02)$ at the intervention versus the control site. Preand postimplementation, a score $>5$ was positively associated with abnormal test results (OR $=4.0$; 95\% CI: $1.8-9.2)$ and trainee provider $(\mathrm{OR}=2.3 ; 95 \% \mathrm{CI}: 1.2-4.6)$. Postimplementation, a score $>5$ was also positively associated with intervention site $(\mathrm{OR}=2.3 ; 95 \%$ CI: $1.1-5.1)$ and specialty clinic $(\mathrm{OR}=2.0 ; 95 \% \mathrm{CI}: 1.1-3.6)$. There were also significantly fewer tests ordered after implementation (264/100,000 vs. $204 / 100,000 ; P=0.03$ ), with no significant change at the control site $(280 / 100,000$ vs. $257 / 100,000 ; P=0.50)$.

Conclusions: EHR point-of-care tools improved documentation of genetic testing processes and decreased utilization of genetic tests commonly ordered by nongeneticists.

Genet Med advance online publication 30 June 2016

\section{INTRODUCTION}

Genetic testing has become more accessible and relevant to every medical specialty. ${ }^{1,2}$ However, there are concerns that the health-care workforce is not prepared to meet the challenge of integrating genetics into clinical practice, which could result in inappropriate use of genetic tests and related services, ${ }^{3-8}$ potentially leading to medical errors. ${ }^{9}$ Inappropriate use of genetic testing has been attributed to inadequate care processes (e.g., insufficient collection of personal and family history needed to inform a differential diagnosis, test selection strategy, informed consent, and management options) as well as clinicians' lack of knowledge about genetic principles, testing methods, and their limitations. ${ }^{3-8}$

Processes of care are described in medical record documentation. Medical record documentation facilitates diagnosis and treatment, communicates pertinent information to other health-care providers to ensure patient safety and reduce medical errors, and serves as an important medical-legal function in risk management. ${ }^{10}$ The National Committee for Quality Assurance has created standards for medical record documentation that reflect the importance of complete, timely, and accurate medical record documentation. For laboratory and other diagnostic tests, results should be documented and reviewed by the ordering clinician, and abnormal laboratory results should be conveyed to the patient and have an explicit notation in the record describing follow-up plans or changes in treatment. ${ }^{11}$

Common HFE mutations, factor V Leiden, and HLA-B27 are examples of genetic tests whereby best practices in processes of care are often not followed. ${ }^{12-17}$ HFE testing is used to support the diagnosis of hereditary hemochromatosis in patients with evidence of iron overload; however, only $50 \%$ of individuals have evidence of iron overload at the time of HFE testing. ${ }^{12,13}$ In a large nonprofit health-care system, only $37 \%$ of factor V Leiden tests ordered met the internal guideline of the health-care system, $46 \%$ met the College of American Pathologists' guideline, and $61 \%$ met the American College of Medical Genetics and

${ }^{1}$ VA Greater Los Angeles Healthcare System, Los Angeles, California, USA; ${ }^{2}$ Department of Medicine, David Geffen School of Medicine at UCLA, Los Angeles, California, USA; ${ }^{3}$ Department of Psychiatry and Biobehavioral Sciences, David Geffen School of Medicine at UCLA, Los Angeles, California, USA; ${ }^{4}$ Department of Pathology and Laboratory Medicine, David Geffen School of Medicine, UCLA, Los Angeles, California, USA; ${ }^{2}$ Quest Diagnostics, Madison, New Jersey, USA; ${ }^{6}$ Department of Health Policy and Management, Fielding School of Public Health, UCLA, Los Angeles, California, USA; ${ }^{7}$ Centers for Disease Control and Prevention, Atlanta, Georgia, USA. Correspondence: Maren T. Scheuner (maren.scheuner@va.gov) 
Genomics' guideline. ${ }^{15}$ HLA-B27 is frequently present in axial spondyloarthritis, but it has been shown to have limited utility as a diagnostic test, especially in the hands of primary-care clinicians and particularly in populations with low prevalence of HLA-B27. ${ }^{15-17}$

Approaches to improve genetic testing care processes and related health-care outcomes include clinician education and changes to organizational structures and procedures, such as guidance for requisition of genetic tests, use of a standardized laboratory report, integration of genetics-related clinical decision support in the electronic health record (EHR), and increasing access to genetics professionals or Internet resources. ${ }^{9,18}$ The goal of this study was to evaluate the impact of EHR point-ofcare tools on medical record documentation of genetic testing care processes for the common HFE mutations, a thrombophilia panel, and HLA-B27.

\section{MATERIALS AND METHODS}

\section{Study design}

We conducted a nonrandomized, controlled, pre-/postintervention study. The intervention targeted genetic tests that were orderable in the EHR at the intervention and control sites, including the HFE C282Y and H63D mutations, a thrombophilia panel (factor V Leiden, prothrombin G20210A variant, and MTHFR C677T and A1298C variants), and HLA-B27. There were three study phases: a 6-month preimplementation period of data collection without intervention (April 2011 to September 2011); a 15-month implementation period (April 2012 to June 2013) during which the intervention for each genetic test was introduced sequentially in the first 3 months, with 12 months of implementation for each test; and a 6-month postimplementation data collection phase (July 2013 to December 2013). We used mixed methods, including chart review, focus groups, and interviews. The Institutional Review Board at the Veterans Affairs (VA) Greater Los Angeles Healthcare System approved all study procedures.

Two VA health-care systems served as the intervention and control sites. Each health-care system comprises a large, highcomplexity VA medical center and its respective communitybased outpatient clinics. Both sites are in southern California, both are served by the same Veteran Integrated Service Network and network-based laboratory, and both are affiliated with local medical schools and provide medical training.

\section{Frameworks}

The Centers for Disease Control and Prevention has developed a framework distinguishing three phases in the "total genetic testing process": a preanalytic phase comprising processes of care before a test is performed, an analytic phase comprising laboratory activities, and a postanalytic phase that consists of care processes after the test result is available. ${ }^{19}$ Characterizing the genetic testing process in this way provides a framework for assessing genetic testing-related processes of care. We used this framework to inform the primary outcomes and data collection for this project. We used the domains and constructs from the
Consolidated Framework for Implementation Research most relevant to provider behavior, including provider characteristics, patient characteristics, internal organizational structures and procedures, and external factors (e.g., guidelines, best practices) to evaluate themes identified from the qualitative results and to synthesize findings from both the qualitative and quantitative results. ${ }^{20}$

\section{Intervention}

The intervention included point-of-care tools in the EHR and didactic lectures and information resources to educate providers on use of these tools. The EHR point-of-care tools included (i) informational messages at the time of test ordering and (ii) synoptic test reports generated as a progress note using a template. The informational messages reminded clinicians to document the reason for testing and informed consent and, for HFE testing, to document evidence of iron overload (i.e., iron/total iron-binding capacity (TIBC) $\times 100>45 \%$ ) or known family history of hereditary hemochromatosis, in addition to informed consent. These messages were not hard stops in the ordering process; a clinician could choose to ignore them and continue ordering the test. For the synoptic report, we used a template previously shown to improve satisfaction, ease of use, and effectiveness of communicating results..$^{21,22}$ Project team members monitored all of the orders and results for the genetic tests undergoing study at the intervention site, and they generated a report for each test result using the progress note template that had fields for clinical and family history, test name and indication, test result and interpretation, guidance, and supplemental information. The template report was complementary to the standard laboratory report in the laboratory section of the medical record and was available in the EHR usually the same day (and not more than 2 days after) as the posting of the standard laboratory report. The ordering clinician, the attending physician, if indicated, and the primary-care clinician were alerted to sign the template report as a way of acknowledging receipt.

At the intervention site, in the first few months of implementation, eight didactic lectures were given (by M.T.S.) to a variety of clinical audiences on the topic of genetic testing, and information resources were distributed to staff physicians, nurse practitioners and physician assistants, including (i) availability of a clinician-to-clinician genetic consult using the EHR (i.e., a genetics e-consult), (ii) a "chart biopsy" report available in the EHR for each genetic test that pulls relevant data from a patient's record (e.g., whether the test has been performed previously and related tests and procedures), and (iii) information sheets distributed through e-mail and hard copy describing the availability of a genetic e-consult, the availability of the "chart biopsy," and the importance of documenting the informedconsent process when ordering genetic tests.

\section{Best practices}

For each test ordered, we assessed adherence to best practice. HFE testing is indicated for patients with evidence of iron 


\section{ORIGINAL RESEARCH ARTICLE}

overload or a family history of hereditary hemochromatosis. ${ }^{23,24}$ We considered best practice for HFE testing when there was a relevant family history or when iron studies (i.e., iron, TIBC, and/or ferritin) were ordered prior to HFE testing. Because we could not assess iron study results, we probably overestimated best practices. Thrombophilia panel testing is indicated for patients with thromboembolism before age 50 years or when there is a family history of thromboembolic disease or a known thrombophilia mutation. ${ }^{25,26}$ We could not differentiate between incident or recurrent thromboembolic events; therefore, we may have underestimated best practice because patients with recurrent events at any age are candidates for testing. However, only $15 \%$ of individuals with a thromboembolic event will have a recurrent event, and usually this will occur at younger ages. ${ }^{27}$ HLA-B27 testing is indicated to assess the etiology of low back pain or anterior uveitis. For anterior uveitis, no additional studies are necessary prior to testing. ${ }^{28,29}$ For low back pain, imaging of the sacroiliac joints for evidence of ankylosing spondylitis should occur prior to HLA-B27 testing; when there are normal results, HLA-B27 testing might help in elucidating the cause of the back pain. We considered it best practice when sacroiliac imaging was ordered prior to HLA-B27 testing for all indications other than anterior uveitis. We could not consider the results of sacroiliac imaging; thus, we probably overestimated best practice. However, most (90\%) imaging studies will be normal..$^{28,29}$

\section{Quantitative materials and methods}

Chart review was used to collect the main outcomes of interest. Records from outpatients who had one of the genetic tests ordered by a nongeneticist during the 6-month period before implementation or during the 12 months after implementation were eligible. For every record, a chart summary comprising structured and text-based data was generated as an electronic Microsoft Word file. The structured data included the name of the genetic test and results, the site, the specialty and type of the ordering provider (physician, nurse practitioner, physician assistant or trainee), patient demographics, and active problem list. Text-based data included all progress notes written by a nongeneticist (i.e., the template report was excluded) in the 6 months prior to the test order (preanalytic phase) and 6 months after the test results (postanalytic phase). All HIPAA identifiers were redacted prior to sharing the chart summaries with the research team.

Once the redacted chart summaries were available, independent double-coding of each record was performed using ATLAS.ti (version 6; Scientific Software Development, Berlin, Germany) to assist with recognition of documented care processes through key words and phrases with adjudication of discrepancies by a third researcher and input from the principal investigator (M.T.S.) when needed. In each record, we assessed documentation in the preanalytic and postanalytic phases of genetic testing. For each documentation item, we collected the date and author of the note, specifying ordering or nonordering provider, specialty, and type of clinician. Coded data were dually entered into a Microsoft Access database, and a third researcher resolved data entry discrepancies with input from the research team as needed. Descriptions of the development and quality assurance for this method are available in the Supplementary Material online.

\section{Analyses}

For each documentation item, we assigned a score depending on the completeness of documentation (as shown in Table 2 with examples provided in the table legend), with a maximum score of 12 points. The main outcome measure was the mean total sum of points in each record. With a sample size of 240 at the intervention site and 300 at the control site, we estimated we could detect a delta of 0.5 points for $80 \%$ power and a delta of 0.6 points for $90 \%$ power. Another outcome measure was a documentation score $>5$, which was considered clinically meaningful because a score of 5 was the mean value at baseline and a score of 6 or more would require documentation in both the preanalytic and postanalytic phases of genetic testing. We used multiple linear and logistic regressions to assess associations between the documentation score (a mean score and score $>5$ ) and patient, provider, and test characteristics. Variables included the following: patient demographics, number of active medical problems, and number of progress notes per record; genetic test name, reason for testing, and normal or abnormal test results; and ordering provider characteristics. All analyses were conducted using STATA (Stata Statistical Software: Release 13, StataCorp, College Station, TX).

\section{Qualitative materials and methods}

During the preimplementation period, we recruited clinicians who had ordered at least one genetic test of interest at the intervention or control site in the prior 12 months to participate in a phone or in-person interview or focus group. The objective was to understand the current process of ordering and receiving test results and learning how best to implement our planned interventions. To obtain feedback on the individual components of the intervention and barriers and facilitators to implementation, we conducted interviews with clinicians at the intervention site who had ordered at least one genetic test of interest during the 6 to 7 months after implementation. At the time of recruitment, clinicians were informed of the study purpose; at the time of participation, clinicians gave consent. Clinicians with specialty training in medical genetics were not eligible.

\section{Qualitative analyses}

Interviews were recorded and transcribed verbatim. Transcripts were summarized in a matrix according to key domains of interest to provide rapid feedback to the research team. More detailed analysis of the transcripts followed using ATLAS.ti. Key domains and subdomains constituted the initial deductive code list. The principal investigator (M.T.S.) reviewed the coding output to ensure relevant domains were being captured. Two coders independently coded a portion of transcripts, and then coding was compared to examine the degree of consensus. 
Lack of consensus was resolved through discussion and adjustments were made to the code list. Additional inductive codes were added to the code list as needed.

\section{RESULTS}

\section{Chart review}

During the 6-month preimplementation period, an estimated 41,995 unique patients were seen at the intervention site and 32,500 were seen at the control site. During the 12 -month postimplementation period, an estimated 85,639 unique patients were seen at the intervention site and 67,858 were seen at the control site. ${ }^{30-32}$ We reviewed 202 records (111 at the intervention site and 91 at the control site) of patients who had a genetic test in the 6-month preimplementation period and 349 records (175 intervention and 174 control site) of patients who had a genetic test during the 12-month postimplementation period. There was a significant pre-/postimplementation decrease in the number of tests ordered at the intervention site $(264 / 100,000$ vs. $204 / 100,000$, respectively; $P=0.03$ ), without significant pre-/postchange decrease at the control site $(280 / 100,000$ vs. 257/100,000, respectively; $P=0.5$ ).

Table 1 shows the characteristics of the patients who had a genetic test and the ordering clinicians. The intervention site had younger and fewer white patients; almost $50 \%$ of ordering clinicians were trainees compared with only one-third at the control site. A little more than $60 \%$ of the tests were ordered in specialty clinics, and the remaining were ordered in primarycare clinics (internal medicine, ambulatory care, and women's health) at both sites. There were no site differences according to the number of tests ordered by a clinician, with the majority ordering a test only once.

The documentation of the genetic testing care process at the intervention and control sites is shown in Table 2. Documentation of the test results improved significantly at the intervention site before and after implementation, with increases in documentation of both general (5.8 vs. $10.9 \%$, respectively) and specific (47.7 vs. $54.9 \%$, respectively) results $(P=0.03)$. There was also a significant increase in documentation of the implications of test results at the intervention site compared with the control site ( 37.7 vs. $29.3 \%$, respectively; $P=0.02)$.

Table 3 shows the percentage of records with a score of 0 to 12 in the pre- and postimplementation periods. There were no differences in the mean documentation score at baseline between sites $(5.2(\mathrm{SD}=2.1)$ vs. $5.2(\mathrm{SD}=2.2))$. After implementation, we observed a significant increase in the mean documentation score at the intervention site $(5.9(\mathrm{SD}=2.1)$ vs. $5.0(\mathrm{SD}=$ $2.2) ; P=0.0001$ ). There was no significant pre-/postdifference in the frequency of records with a score $>5$ at the control site ( 48 to $46 \% ; P=0.73$ ); however, at the intervention site, we saw a significant increase in records with a score $>5$ (47 to $59 \%$; $P=0.02)$.

Across all records, during the pre- and postimplementation periods, a score $>5$ was associated with abnormal test results $(\mathrm{OR}=4.0 ; 95 \% \mathrm{CI}: 1.8-9.2)$ and when tests were ordered by a trainee instead of a staff physician (OR $=2.3$; 95\% CI: $1.2-4.6)$. After implementation, a score $>5$ was positively associated with the intervention site versus the control site $(\mathrm{OR}=2.3$; 95\% CI: 1.1-5.1) and specialty- versus primary-care clinic $(\mathrm{OR}=2.0$; 95\% CI: 1.1-3.6). Also, Hispanic ethnicity of the patient was negatively associated with a score $>5$ when compared with nonHispanic whites $(\mathrm{OR}=0.3$; 95\% CI: 0.2-0.6); no other racial differences were observed. We observed comparable associations using total scores.

\section{Best practices}

There were no significant site differences in frequency of best practices overall or for each of the genetic tests. For all tests combined, we found that $41 \%$ of records had evidence of best practices prior to implementation at both sites. Postimplementation, there was a $6 \%$ increase in best practices at the intervention site versus the control site ( 47 vs. $41 \% ; P=0.33$ ). Factors associated with best practices across all records in both the pre- and postimplementation periods included the following: younger patient age $(\mathrm{OR}=0.97 ; 95 \% \mathrm{CI}$ : 0.96-0.99; $P<0.001)$; female gender $(\mathrm{OR}=2.1 ; 95 \% \mathrm{CI}: 1.0-4.1, P=0.04)$; and type of test, with more frequent best practices for thrombophilia $(\mathrm{OR}=2.4 ; 95 \% \mathrm{CI}: 1.5-3.8 ; P<0.001)$ and HFE testing (OR $=6.3$; 95\% CI: 3.7-10.7; $P<0.001)$ compared with HLA-B27 testing. Intervention versus control site, patient race/ethnicity, number of active medical problems, number of progress notes, and type or specialty of ordering provider did not influence best practices.

\section{Preimplementation focus groups and interviews}

We interviewed 10 clinicians at the intervention site and 9 clinicians at the control site. We held two focus groups with 4 and 3 clinicians at each from the intervention site and control site, respectively. Participation in an interview or focus group was arranged according to provider preference. At the intervention site, $64 \%$ were women and $64 \%$ were primarycare clinicians; at the control site, half were women and $67 \%$ were primary-care clinicians. Participants reported that the standard existing genetic test reports were not particularly helpful with clinical decision making and were often confusing. Test results were not always reviewed if normal, and no alert was automatically sent to the provider if the results were normal. Additionally, when trainees order tests, the attending provider would not be alerted about the results. Based on these findings, during the implementation period, we identified the ordering provider, attending physician (if ordering provider was a trainee), and the primary-care clinician as signers of the template report.

\section{Midimplementation interviews}

We interviewed 16 clinicians who had ordered a genetic test at the intervention site 6 to 7 months after implementation. Half were women and 56\% were primary-care clinicians. Table 4 describes recall and reactions to each component of the intervention. Overall, the template report was most influential. 
Table 1 Characteristics of records from patients having a genetic test of interest at the control and intervention sites during the pre- and postimplementation periods

\begin{tabular}{|c|c|c|}
\hline Patient characteristics & $\begin{array}{c}\text { Control site } \\
(n=265)\end{array}$ & $\begin{array}{l}\text { Intervention } \\
\text { site }(n=286)\end{array}$ \\
\hline Male, \% & 96.0 & 88.6 \\
\hline \multicolumn{3}{|l|}{ Patient age (years) mean, SD } \\
\hline Male $^{a}$ & $57.0 \pm 14.1$ & $54.4 \pm 14.8$ \\
\hline Female & $44.8 \pm 16.9$ & $41.4 \pm 11.5$ \\
\hline \multicolumn{3}{|l|}{ Race/ethnicity, \% ${ }^{\mathrm{b}}$} \\
\hline White & 53.2 & 40.2 \\
\hline African American & 15.1 & 24.8 \\
\hline Hispanic & 16.6 & 13.6 \\
\hline Asian, Am Indian, Pac Islander, Mixed & 4.2 & 5.6 \\
\hline Declined to answer & 10.9 & 15.7 \\
\hline $\begin{array}{l}\text { No. of active medical problems } \\
\text { (median, range) }\end{array}$ & $12,0-45$ & $11,0-42$ \\
\hline $\begin{array}{l}\text { No. of progress notes per record } \\
\text { (median, range) }\end{array}$ & $50,4-789$ & $45,1-624$ \\
\hline \multicolumn{3}{|l|}{ Genetic test characteristics } \\
\hline \multicolumn{3}{|l|}{ Test of interest, $\%^{c}$} \\
\hline HFE C282Y, H63D & 35.5 & 21.3 \\
\hline Thrombophilia panel $^{d}$ & 33.2 & 38.5 \\
\hline HLA-B27 & 31.3 & 40.2 \\
\hline \multicolumn{3}{|l|}{ Reason for testing, \% ${ }^{\mathrm{e}}$} \\
\hline Diagnostic & 95.9 & 96.9 \\
\hline Predisposition & 3.4 & 2.1 \\
\hline \multicolumn{3}{|l|}{ Test results, \% } \\
\hline Abnormal $^{\dagger}$ & 25.3 & 22.4 \\
\hline Normal & 74.3 & 75.5 \\
\hline Not available (canceled or inconclusive) & 0.4 & 2.1 \\
\hline \multicolumn{3}{|l|}{ Ordering provider characteristics } \\
\hline \multicolumn{3}{|l|}{ Type of ordering provider, $\%^{9}$} \\
\hline Physician & 57.4 & 39.5 \\
\hline Nurse practitioner/physician assistant & 10.2 & 12.2 \\
\hline Trainee $^{h}$ & 32.5 & 48.3 \\
\hline \multicolumn{3}{|l|}{ Specialty, \% ${ }^{i}$} \\
\hline Primary care & 38.1 & 37.2 \\
\hline Specialist & 61.9 & 62.8 \\
\hline \multicolumn{3}{|l|}{ No. of tests ordered (median, range) } \\
\hline 1 & 55.2 & 60.3 \\
\hline $2-4$ & 31.0 & 28.6 \\
\hline $5-10$ & 9.2 & 7.9 \\
\hline 11 or more & 4.6 & 3.2 \\
\hline
\end{tabular}

There were no significant pre-/postimplementation differences at either the intervention site or the control site.

Am, American Indian; Pac, Pacific.

${ }^{\mathrm{a} P}=0.04 .{ }^{\mathrm{b} P}=0.005 .{ }^{\mathrm{c} P}=0.001$. ${ }^{\mathrm{d} F a c t o r} \mathrm{~V}$ Leiden mutation or $\mathrm{APC}$ resistance, factor II G20210A mutation, and MTHFR C677T and A1298C variants. "Reason for testing was inferred by coders. Diagnostic testing was assigned when a patient had signs and symptoms of a genetic diagnosis and predisposition testing when testing was performed given a family history of conditions suspicious for a genetic diagnosis. ${ }^{\mathrm{f}} \mathrm{Abnormal}$ tests included homozygosity, compound heterozygosity or heterozygosity for factor $\mathrm{V}$ Leiden and/or factor II G20210A (intervention site, $n=10$; control site, $n=16$ ), HFE C282Y and/or HFE H63D (intervention site, $n=29$; control site, $n=36$ ), or HLA-B27-positive (intervention site, $n=25$; control site, $n=15)$. ${ }^{9 P}<0.001$. 'Includes medical student, intern, resident, and fellow. ispecialty was determined by signature block or progress note title. Primary care included ambulatory care, women's health and internal medicine. Specialty included cardiology, gastroenterology, hematology-oncology, neurology, ophthalmology, physical medicine and rehabilitation service, rheumatology, and other specialties.

\section{DISCUSSION}

Laboratory testing is an integral part of many medical decisions, providing clinicians with critical information necessary for disease prevention, diagnosis, treatment, or management.

Through implementation of point-of-care tools in the EHR, we found improved documentation of the processes of care for genetic tests commonly ordered by nongeneticists. As described in the literature, the quality of documentation may reflect the quality of care delivered ${ }^{33}$; therefore, these interventions have the potential to improve outcomes related to integration of genetic test results in care delivery.

Clinicians identified the template report as the most memorable and influential intervention, and most improvements in documentation were observed in the postanalytic phase of genetic testing, which probably reflects the influence of the template genetic test reports. Because the majority of clinicians had ordered a genetic test only once during the study period, there was limited opportunity for the template report to influence test orders or documentation of preanalytic processes of care in most cases. Over time, as clinicians receive more genetic tests results with this report format, it is possible that the template report could influence preanalytic phase documentation of care processes for subsequent tests. The informational messages at the time of test ordering were also described as useful but not memorable, and they could have a bigger impact if translated to hard stops in the test ordering process. Lectures providing information about the genetic testing process were also memorable and described as useful, whereas this was not the case for e-mail messages about genetic testing best practices or the availability of e-consults.

We observed significantly fewer tests ordered at the intervention site postimplementation, indicating effects of the intervention on decisions to order a genetic test. Review of the literature has shown that these tests tend to be overutilized. However, we could not assess appropriateness for patients who should have been tested but were not tested, and we did not observe significant improvements in best practices at the intervention site. The decrease in test orders at the intervention site may have been due to the pop-up informational messages at the time of test ordering. Prior studies of computer decision support or information display for curtailing unnecessary or redundant tests or increasing the appropriateness of tests and their timing have had similar modest positive impacts on diagnostic, screening, or monitoring test ordering. ${ }^{34,35}$ Alternatively, clinicians may have been dissuaded from ordering subsequent genetic tests after receiving and reviewing the template report. The lectures and distribution of information resources might have also influenced test ordering at the intervention site.

Factors associated with best practices regarding ordering of the genetic tests under study included patient characteristics (younger age, female gender) and type of test, with best practices occurring more often for HFE, followed by thrombophilia and HLA-B27 testing. The strength of evidence and availability of evidence-based guidelines may have 
Table 2 Documentation by site during the pre- and postimplementation periods

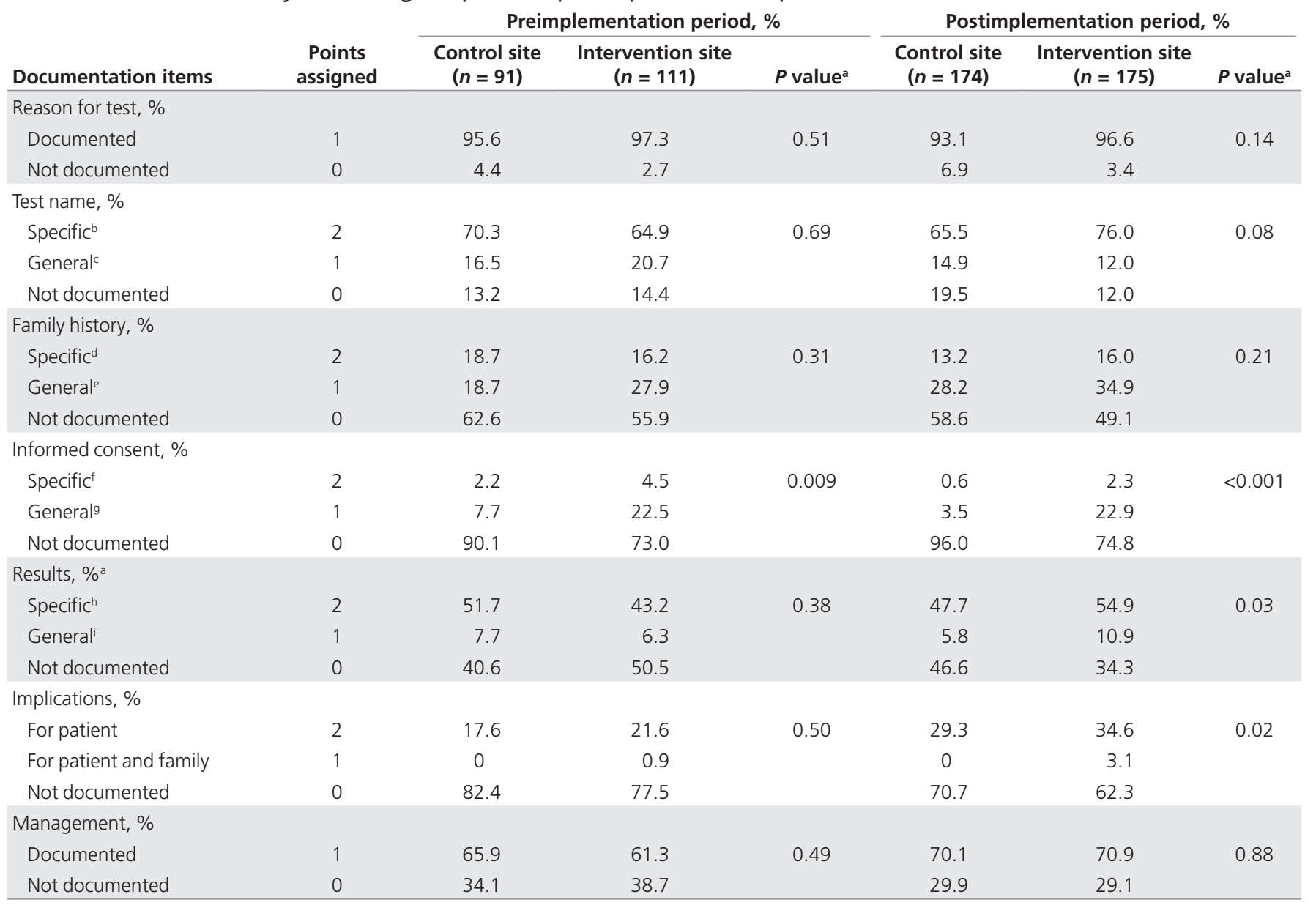

aChi-squared analysis using Fisher's exact test with a two-tailed Pvalue. "'Specific documentation for test name examples: For HFE, "HFE genetic screen." "Elevated LFTs_order ESR, CRP, ANA, smooth muscle Ab, hemochromatosis panel, ceruloplamin (R/O Wilson's), alpha-1 antitrypsin..." "High ferritin, hemochromatosis today." For thrombophilia, "WILL CK FOR 'HYPERCOAG STATE' (LEIDEN FACTOR, ETC.....)." "Will check factor V Leiden, factor VIII, and factor IX levels, prothrombin gene mutation, beta-2-glycoprotein level, anticardiolipin ab, lupus anticoagulant, antithrombin III." For HLA, "Will send HLA B27." "Check HLA b27." "Check labs today, ENA panel, HLA b27..." 'General documentation for test name examples: For HFE, "Iron overload...labs today." "Labs: iron studies." For thrombophilia, "Hypercoaguable work up will be done as outpatient." "Hypercoag labs ordered." "Presenting to hematology to discuss hypercoag work up." For HLA, "Check labs for rheumatologic causes, re-check esr, crp, cbc/diff..." dSpecific documentation for family history examples: For HFE, "Family history of hemochromatosis on mother's side." "Patient has two family members that passed away from liver failure 2/2 hemochromatosis." For thrombophilia, "Family hx: is noted for many relatives with thromboembolic disease. Mother had DVT in her 50s, pt thinks in legs. Sister died suddenly (age 60s) while attending the funeral of a different sister, thought to be PE. She had been diagnosed with clotting disorder prior to this but was not taking her Coumadin." "No family hx of hypercoagulable d/o." For HLA, "No known family history of autoimmune disease, psoriasis, spondyloarthropathy." eGeneral documentation for family history examples: For HFE, "Family history: mother—diabetes, 1 uncle who died of liver failure." "Denies fam hx liver d." For thrombophilia, "FHX: DM" "Mother: HTN, DM, died of MI at 58." For HLA, "No autoimmune diseases." "Family history: not sure if RA/lupus/autoimmune dx or CA." "FH: No known rheumatological disorder." "Specific documentation for informed-consent examples: For HFE, "I discussed with him the need for further blood tests (hemochromatosis mutation analysis)." For HLA, "risks and benefits of such genetic testing discussed with pt and how a positive test would not necessarily rule in a spondyloarthropathy and how a negative test would not rule out a spondyloarthropathy; pt understands these features of HLA-B27 testing and testing is ordered." "General documentation for informed-consent examples: "patient verbalizes understanding and consents to the treatment plan outlined above." "Today's diagnostic and treatment plans were discussed with the patient who was given an opportunity to ask questions and who expressed his understanding of and agrees with the plans to manage his rheumatic condition." "Pt was appropriately counseled regarding diagnostic and therapeutic plans and medications. Pt expressed understanding and agreement." "hpecific documentation for test result examples: "HFE positive," "Hemochromatosis gene negative," "FVL carrier," "HLAB27 positive," HLA-B27 negative." 'General documentation for test result examples: For HFE, "Iron studies normal"; For thrombophilia, "clotting work up normal"; For HLA, "Rheum w/u nl."

contributed to the observed differences in best practices by test type, with a stronger evidence base for HFE and thrombophilia testing than for HLA-B27 (refs. 25-29). Younger patient age is not a surprising finding because younger age of disease onset is a feature of genetic predisposition for most adult-onset conditions, and younger age is a consideration for ordering HFE, thrombophilia, and HLA-B27 testing. ${ }^{25-29}$ Women generally know and report a family history more often than their male counterparts, ${ }^{36}$ and this may explain the increased frequency of genetic testing best practices for female patients.

Certain clinician, patient, and test characteristics were significantly associated with documentation of genetic testing processes of care. Documentation was strongly associated with abnormal test results both before and after implementation, and this is expected given the important implications of such results. Both before and after implementation, trainees were significantly more likely to document genetic testing process of 
care. This may be due to the relative novelty of ordering these genetic tests as compared with the staff clinicians, for whom ordering may have become routine. After implementation, specialists were more likely than primary-care clinicians to

Table 3 Documentation scores by site during the pre- and postimplementation periods

\begin{tabular}{lccccc} 
& \multicolumn{2}{c}{$\begin{array}{c}\text { Preimplementation } \\
\text { period, \% }\end{array}$} & & \multicolumn{2}{c}{$\begin{array}{c}\text { Postimplementation } \\
\text { period, \% }\end{array}$} \\
\cline { 2 - 3 } \cline { 5 - 6 } Score & $\begin{array}{c}\text { Control site } \\
(\boldsymbol{n}=\mathbf{9 1})\end{array}$ & $\begin{array}{c}\text { Intervention } \\
\text { site }(\boldsymbol{n}=\mathbf{1 1 1})\end{array}$ & $\begin{array}{c}\text { Control site } \\
(\boldsymbol{n}=\mathbf{1 7 4})\end{array}$ & $\begin{array}{c}\text { Intervention } \\
\text { site }(\boldsymbol{n}=\mathbf{1 7 5})\end{array}$ \\
\hline 0 & 2.2 & 0 & & 1.7 & 0.6 \\
\hline 1 & 5.5 & 1.8 & & 5.8 & 0.6 \\
\hline 2 & 4.4 & 7.2 & & 6.9 & 2.9 \\
\hline 3 & 11.0 & 18.0 & & 12.6 & 9.7 \\
\hline 4 & 13.2 & 17.1 & & 16.1 & 14.9 \\
\hline 5 & 15.4 & 9.9 & & 10.3 & 12.6 \\
\hline 6 & 18.7 & 16.2 & & 17.2 & 19.4 \\
\hline 7 & 16.5 & 15.3 & & 16.7 & 15.4 \\
\hline 8 & 7.7 & 9.9 & & 9.2 & 14.3 \\
\hline 9 & 5.5 & 1.8 & & 2.9 & 8.0 \\
\hline 10 & 0 & 1.8 & & 0.6 & 0.6 \\
\hline 11 & 0 & 0.9 & & 0 & 0.6 \\
\hline 12 & 0 & 0 & & 0 & 0.6 \\
\hline Mean, SD & $5.2,2.2$ & $5.2,2.1$ & & $5.0,2.2$ & $5.9,2.1^{\text {a }}$ \\
\hline
\end{tabular}

${ }^{a} P=0.0001$ comparing postimplementation intervention to control sites. document outcomes, and this might be related to specialists having a greater awareness and knowledge about the these genetic tests, ${ }^{37}$ which may have facilitated acceptance and integration of the interventions into their practice. We found decreased documentation of genetic testing outcomes for Hispanic patients compared with whites after implementation; this may be due to greater awareness of the lower prevalence and relevance of the genetic tests of interest in this population, ${ }^{38-40}$ resulting in a reduced confidence in documenting implications of the test results-especially normal results-for Hispanics.

\section{Limitations \\ We used a quasi-experimental design because randomization was not feasible. To guard against threats to internal validity, we included a control site that was similar to the intervention site in location, size, complexity, and number of genetic tests ordered. We controlled for important confounding variables in our analyses and we included pre-/postimplementation com- parisons to account for temporal trends. We assessed processes of care only for tests that were ordered. We did not assess care processes for patients who should have been tested but were not tested. Although the VA is one of the largest integrated health- care systems in the United States and our findings are probably relevant to those of other VA facilities, our findings may not be relevant to other settings, particularly solo or small group prac- tices. Importantly, the strategies we used could only be effective}

Table 4 Midimplementation interviews: intervention recall and reactions from 16 clinicians at the intervention site $\begin{array}{lc}\text { Intervention } & \text { Recall prior to being shown } \\ \text { component } & \text { example }\end{array}$

EHR Three recalled seeing one of the EHR

Announcement Announcements by e-mail; another

e-mail

Genetic

e-consult

Lecture on genetic testing

Informational

message

Genetic

Nine recalled seeing the template report

template report
Seven recalled attending a genetics

lecture

Two recalled seeing the pop-up messages when ordering a genetic test
Most providers do not read the EHR Announcements. Reason: too much e-mail. Only 4 thought EHR Announcements could be an effective way to inform providers about genetics topics.

Most thought the e-consult could be a useful tool; good in situations where they are not sure if a test is indicated or need help interpreting results. One remembered using the consult and said it was too burdensome to complete: "As a busy physician I never wanted to do it again."

All found the lecture useful to understanding the genetic testing process

Most thought a message at the point of care was an effective way to communicate information about a genetic test. However, some voiced concerns that these messages could be too easily overlooked and appeared too late in the process after the decision to order a test had been made. As one primary-care clinician said, "I would ignore the info message unless it was a hard stop."

Overall, the most influential component. The general layout and organization were viewed favorably: "If this is the way that you guys are going to be implementing the new way of doing the genetic reporting, I like it"; "The way it's organized, it seems to be kind of incremental so the most important is placed first and then if you're interested in more, you can keep reading...that's the way it should be." Clinicians noted that the organization facilitated their ability to find and read sections of greatest interest. Nearly all agreed the report contained a considerable amount of text; yet only a few found it to be burdensome and distracting. Most felt that the report provided more detail than the standard

laboratory report and provided a clearer explanation of the results and interpretation. "The one from the lab just tells you if it's detected or not detected, and the note goes on to explain what that means." Most said the report would help in explaining the results to the patient. Some said they wanted the report to be more directive and to have more specific recommendations regarding management and implications for family members. Most found that the report helped in understanding the limitations of the test result. Five thought that being alerted to sign the report was helpful. 
in a health-care system like the VA, which has an EHR with clinical decision support capabilities.

In summary, implementation of point-of-care tools in the EHR increased and improved documentation of the processes of care for genetic tests commonly ordered by nongeneticists, suggesting the potential to improve outcomes related to better integration of genetic test results in care delivery. The study interventions most likely influenced clinicians who were willing to learn and change their habits regarding genetic testing and documentation of related care processes. Complementary strategies that could improve test orders and integration of test results include policies that incentivize clinicians, such as performance measures or improved reimbursement for adhering to best practices. Research is needed to understand the importance of factors facilitating or hindering appropriate genetic test ordering and utilization of test results. It will also be important to show improved outcomes as a result of these interventions.

\section{SUPPLEMENTARY MATERIAL}

Supplementary material is linked to the online version of the paper at http://www.nature.com/gim

\section{ACKNOWLEDGMENTS}

This study was funded by the Centers for Disease Control and Prevention Laboratory Training, Evaluation, and Quality Assurance Programs (CDC-RFA-Cl10-100503CONT12; grant 5U47CI000823). This publication was supported by a cooperative agreement from the Centers for Disease Prevention and Control. E.M.Y.'s work was supported by a VA HSR\&D Senior Research Career Scientist Award (RCS 05-195). The authors thank the VA staff who supported this project, including the following research team members: Albert Aparicio, Nui Brown, Austin Jones, Erica Ma, Russell Madison, Brian Tran, and Claudia Vaughn. The authors also thank the following members of the Advisory Board: Joanne Armstrong, Hawazin Faruki, W. Andrew Faucett, Matthew Goetz, Wayne Grody, Elaine Lyon, Laurence Meyer, Victoria Pratt, Sue Richards, Paul Schneider, Indira Subramanian, Tracy Trotter, Vickie Venne, Ari Weinreb, and Marc Williams.

The findings and conclusions in this article are those of the author(s) and do not necessarily represent the views of the Department of Veterans Affairs or the Centers for Disease Control and Prevention/Agency for Toxic Substances and Disease Registry.

\section{DISCLOSURE}

The authors declare no conflict of interest.

\section{REFERENCES}

1. Manolio TA, Chisholm RL, Ozenberger B, et al. Implementing genomic medicine in the clinic: the future is here. Genet Med 2013;15:258-267.

2. Scheuner MT, Marshall N, Lanto A, et al. Delivery of clinical genetic consultative services in the Veterans Health Administration. Genet Med 2014;16:609-619.

3. Scheuner MT, Sieverding P, Shekelle PG. Delivery of genomic medicine for common chronic adult diseases: a systematic review. JAMA 2008;299: 1320-1334.

4. Burke W, Culver J, Pinsky L, et al. Genetic assessment of breast cancer risk in primary care practice. Am J Med Genet A 2009;149A:349-356.

5. Brierley KL, Campfield D, Ducaine W, et al. Errors in delivery of cancer genetics services: implications for practice. Conn Med 2010;74:413-423.
6. Levy $D E$, Byfield $S D$, Comstock $C B$, et al. Underutilization of BRCA1/2 testing to guide breast cancer treatment: black and Hispanic women particularly at risk. Genet Med 2011;13:349-355.

7. Dhar SU, Cooper HP, Wang T, et al. Significant differences among physician specialties in management recommendations of BRCA1 mutation carriers. Breast Cancer Res Treat 2011;129:221-227.

8. Plon SE, Cooper HP, Parks B, et al. Genetic testing and cancer risk management recommendations by physicians for at-risk relatives. Genet Med 2011;13: 148-154.

9. Zhi M, Ding EL, Theisen-Toupal J, Whelan J, Arnaout R. The landscape of inappropriate laboratory testing: a 15-year meta-analysis. PLOS One 2013:8:e78962.

10. Wood DL. Documentation guidelines: evolution, future direction, and compliance. Am J Med 2001;110:332-334.

11. National Committee for Quality Assurance. Guidelines for medical record documentation. http://www.ncqa.org/portals/0/policyupdates/supplemental/ guidelines_medical_record_review.pdf, Accessed 27 March 2016.

12. Lanktree MB, Lanktree BB, Paré G, Waye JS, Sadikovic B, Crowther MA. Examining the clinical use of hemochromatosis genetic testing. Can J Gastroenterol Hepatol 2015;29:41-45.

13. Adams PC. Epidemiology and diagnostic testing for hemochromatosis and iron overload. Int J Lab Hematol 2015;37 Suppl 1:25-30.

14. Laberge AM, Psaty BM, Hindorff LA, Burke W. Use of Factor V Leiden genetic testing in practice and impact on management. Genet Med 2009;11: 750-756.

15. Lane SK, Gravel JW Jr. Clinical utility of common serum rheumatologic tests. Am Fam Physician 2002;65:1073-1080.

16. Brandt HC, Spiller I, Song IH, Vahldiek JL, Rudwaleit M, Sieper J. Performance of referral recommendations in patients with chronic back pain and suspected axial spondyloarthritis. Ann Rheum Dis 2007;66:1479-1484.

17. Braun A, Gnann H, Saracbasi E, et al. Optimizing the identification of patients with axial spondyloarthritis in primary care-the case for a two-step strategy combining the most relevant clinical items with HLA B27. Rheumatology (Oxford) 2013;52:1418-1424.

18. Lubin IM, McGovern MM, Gibson Z, et al. Clinician perspectives about molecular genetic testing for heritable conditions and development of a clinician-friendly laboratory report. J Mol Diagn 2009;11:162-171.

19. Chen B, Gagnon M, Shahangian S, Anderson NL, Howerton DA, Boone JD; Centers for Disease Control and Prevention (CDC). Good laboratory practices for molecular genetic testing for heritable diseases and conditions. MMWR Recomm Rep 2009;58(RR-6):1-37; quiz CE.

20. Damschroder LJ, Aron DC, Keith RE, Kirsh SR, Alexander JA, Lowery JC. Fostering implementation of health services research findings into practice: a consolidated framework for advancing implementation science. Implement Sci 2009:4:50

21. Scheuner MT, Hilborne L, Brown J, Lubin IM; members of the RAND Molecular Genetic Test Report Advisory Board. A report template for molecular genetic tests designed to improve communication between the clinician and laboratory. Genet Test Mol Biomarkers 2012;16:761-769.

22. Scheuner MT, Edelen MO, Hilborne LH, Lubin IM; RAND Molecular Genetic Test Report Advisory Board. Effective communication of molecular genetic test results to primary care providers. Genet Med 2013;15:444-449.

23. US Preventive Services Task Force. Screening for hemochromatosis: recommendation statement. Ann Intern Med 2006;145(3):204-208.

24. European Association for the Study of the Liver: EASLD clinical practice guidelines for HFE hemochromatosis. J Hepatol 2010;53:3-22.

25. Press RD, Bauer KA, Kujovich JL, Heit JA. Clinical utility of factor V leiden (R506Q) testing for the diagnosis and management of thromboembolic disorders. Arch Pathol Lab Med 2002;126:1304-1318.

26. Spector EB, Grody WW, Matteson CJ, et al. Technical standards and guidelines: venous thromboembolism (Factor V Leiden and prothrombin 20210G >A testing): a disease-specific supplement to the standards and guidelines for clinical genetics laboratories. Genet Med 2005;7:444-453.

27. Marchiori A, Mosena L, Prins MH, Prandoni P. The risk of recurrent venous thromboembolism among heterozygous carriers of factor $\mathrm{V}$ Leiden or prothrombin G20210A mutation. A systematic review of prospective studies. Haematologica 2007:92:1107-1114.

28. van den Berg R, de Hooge M, Rudwaleit M, et al. ASAS modification of the Berlin algorithm for diagnosing axial spondyloarthritis: results from the SPondyloArthritis Caught Early (SPACE)-cohort and from the Assessment of SpondyloArthritis international Society (ASAS)-cohort. Ann Rheum Dis 2013;72:1646-1653 


\section{ORIGINAL RESEARCH ARTICLE}

29. Aydin SZ, Maksymowych WP, Bennett AN, McGonagle D, Emery P, MarzoOrtega $\mathrm{H}$. Validation of the ASAS criteria and definition of a positive MRI of the sacroiliac joint in an inception cohort of axial spondyloarthritis followed up for 8 years. Ann Rheum Dis 2012;71:56-60.

30. Veteran Health Care Made Personal. Annual Report 2011. http://www. desertpacific.va.gov/DESERTPACIFIC/docs/VISN22_AR_2011_508_English_. pdf. Accessed 26 October 2015.

31. VA Desert Pacific Healthcare Network. Personalized Care for our Nation's Heroes. 2012 Annual Report. http://www. desertpacific.va.gov/DESERTPACIFIC/ docs/VISN22_AR_2012_508_English_.pdf. Accessed 26 October 2015.

32. 2013 Annual Report. VA Desert Pacific Healthcare Network. http://www. desertpacific.va.gov/DESERTPACIFIC/docs/VISN22_AR_2013_508_English_. pdf. Accessed 26 October 2015.

33. Peabody JW, Luck J, Glassman P, Dresselhaus TR, Lee M. Comparison of vignettes, standardized patients, and chart abstraction: a prospective validation study of 3 methods for measuring quality. JAMA 2000;283: 1715-1722.

34. Shojania KG, Jennings A, Mayhew A, Ramsay CR, Eccles MP, Grimshaw J. The effects of on-screen, point of care computer reminders on processes and outcomes of care. Cochrane Database of Systematic Reviews 2009; Issue 3. Art. No.: CD001096. doi:10.1002/14651858.CD001096.pub2.

35. Main C, Moxham T, Wyatt JC, Kay J, Anderson R, Stein K. Computerised decision support systems in order communication for diagnostic, screening or monitoring test ordering: systematic reviews of the effects and cost-effectiveness of systems. Health Technol Assess 2010;14:1-227.

36. Scheuner MT, McNeel TS, Freedman AN. Population prevalence of familial cancer and common hereditary cancer syndromes. The 2005 California Health Interview Survey. Genet Med 2010;12:726-735.

37. Vadaparampil ST, Wideroff L, Olson L, Viswanath K, Freedman AN. Physician exposure to and attitudes toward advertisements for genetic tests for inherited cancer susceptibility. Am J Med Genet A 2005;135:41-46.

38. Kujovich JL. Factor V Leiden thrombophilia. Genet Med 2011;13:1-16

39. Steinberg KK, Cogswell ME, Chang JC, et al. Prevalence of C282Y and H63D mutations in the hemochromatosis (HFE) gene in the United States. JAMA 2001;285:2216-2222.

40. Reveille JD, Hirsch R, Dillon CF, Carroll MD, Weisman MH. The prevalence of HLA-B27 in the US: data from the US National Health and Nutrition Examination Survey, 2009. Arthritis Rheum 2012;64:1407-1411. 